GABRIELA MARINI $^{1}$

Jaqueline de CARVALHO RINALDI ${ }^{2}$

DÉbora CRISTINA DamascenO ${ }^{1}$

SÉRGio LuIS FELISBINO ${ }^{2}$

Marilza Vieira Cunha Rudge ${ }^{1}$

Revisão

Palavras-chave

Diabetes mellitus Incontinência urinária Matriz extracelular

Uretra

Fatores de risco

Keywords

Diabetes mellitus Urinary incontinence Extracellular matrix

Urethra

Risk factors

Correspondêncic

Marilza Vieira Cunha Rudge

Distrito de Rubiōo Jr s/n

(EP: 18618-000

Botucatu (SP), Brasil

Recebido

$28 / 04 / 2014$

Aceito com modificaçōes

$24 / 06 / 2014$

DOI: $10.1590 / 50100-720320140005014$

\section{Alterações da matriz extracelular causadas pelo diabetes: o impacto sobre a continência urinária}

\author{
Changes in the extracellular matrix due to diabetes \\ and their impact on urinary continence
}

\section{Resumo}

A prevalência de incontinência urinária em gestantes diabéticas é significantemente elevada e persiste por até dois anos após o parto cesárea, podendo ser a sequela mais frequente da hiperglicemia gestacional comparada a outras complicações. Dessa forma, identificar os fatores de risco para o desenvolvimento da incontinência urinária em diabéticas é o principal objetivo na prevenção dessa condição tão comum. Pesquisas recentes apontam que não apenas o músculo uretral mas também a matriz extracelular uretral desempenham papel importante no mecanismo da continência urinária. Os trabalhos do nosso grupo de pesquisa evidenciaram que, em ratas, o diabetes durante a prenhez lesa a matriz extracelular e o músculo estriado uretral, o que pode explicar a alta prevalência de incontinência e disfunção do assoalho pélvico em mulheres com diabetes mellitus gestacional. $\bigcirc$ diabetes exerce efeito sobre a expressão, organização e alteração dos componentes da matriz extracelular em diversos órgãos, e a remodelação do tecido e a fibrose parecem ser uma consequência direta dele. Assim, a compreensão do impacto de fatores de risco modificáveis, como o diabetes, permitirá que, utilizando estratégias preventivas, reduzamos as taxas de incontinência urinária, bem como os custos de assistência à saúde, e melhoremos a qualidade de vida das mulheres, especialmente na gestação e no pós-parto.

\section{Abstract}

The prevalence of urinary incontinence in diabetic pregnant women is significantly high two years after cesarean section. Incontinence can be the most common consequence of hyperglycemia compared to other complications. Thus, identifying the risk factors for the development of urinary incontinence in diabetes is the major aim in the prevention of this very common condition. Recent surveys have shown that not only muscle but also the urethral extracellular matrix play an important role in the mechanism of urinary continence. Translational work on rats by our research group showed that diabetes during pregnancy damages the extracellular matrix and urethral striated muscle, a fact that may explain the high prevalence of urinary incontinence and pelvic floor dysfunction in women with gestational diabetes mellitus. Diabetes affects the expression, organization and change in extracellular matrix components in different organs, and tissue remodeling and fibrosis appear to be a direct consequence of it. Therefore, understanding the impact of modifiable risk factors, such as diabetes, which involves using preventive strategies, can reduce the rates of urinary incontinence and the health care costs, and improve the quality of life of women, especially during pregnancy and postpartum.
Departamento de Ginecologia e Obstetrícia Faculdade de Medicina de Botucatu, Universidade Estadual Paulista "Julio de Mesquita Filho" - UNESP - Botucatu (SP), Brasil.

'Departamento de Ginecologia e Obstetrícia, Faculdade de Medicina de Botucatu, Universidade Estadual Paulista "Julio de Mesquita Filho" - UNESP - Botucatu (SP), Brasil.

2Departamento de Morfologia, Instituto de Biociências de Botucatu, Universidade Estadual Paulista "Julio de Mesquita Filho" - UNESP Botucatu (SP), Brasil.

Conflito de interesses: não há. 
Introdução

A prevalência de 50,8\% de Incontinência Urinária (IU) em gestantes diabéticas é significantemente elevada e permanece alta por até dois anos após o parto cesárea ${ }^{1}$. Esses achados evidenciam que o diabetes na gestação é fator de risco para o desenvolvimento de IU nas mulheres que não tiveram a influência da via de parto.

A associação entre diabetes e IU é aspecto relevante em termos de saúde pública e de economia em saúde. O mundo vive hoje uma epidemia de diabetes e obesidade, e isso tem influência direta sobre os custos de saúde ${ }^{2}$. A IU é também uma epidemia silenciosa que atinge 200 milhões de pessoas em todo mundo e gerou um custo direto nos Estados Unidos de aproximadamente US $\$ 20$ bilhões de dólares no final do século passado ${ }^{3,4}$.

Os estudos dessa associação são escassos na literatura. Nosso grupo de pesquisa iniciou a pesquisa translacional em ratas prenhes com diabetes de intensidade grave e moderada com Marini et al. ${ }^{5}$ e Piculo et al. ${ }^{6}$, que evidenciaram que o diabetes e a prenhez em ratas danificam o músculo estriado esquelético e a matriz extracelular uretral.

O primeiro trabalho com animais portadores de diabetes grave (glicemia maior que $300 \mathrm{mg} / \mathrm{dL}$ ) demonstrou a ocorrência de adelgaçamento e atrofia do músculo estriado uretral associado à desorganização e rompimento das fibras musculares. A análise imunoistoquímica evidenciou perda da localização anatômica normal das fibras musculares com aumento na proporção de fibras oxidativas 5 .

No segundo trabalho, com o intuito de mimetizar os efeitos do diabetes com elevação moderada da glicemia (glicemia entre 120 e $300 \mathrm{mg} / \mathrm{dL}$ ), verificamos que, além das alterações musculares, podem ser observadas modificações significativas na matriz extracelular, como fibrose, e na ultraestrutura com acúmulo de mitocôndrias, aumento do número de gotas de lipídios e acúmulo de grânulos de glicogênio no grupo diabético prenhe ${ }^{6}$.

As alterações encontradas nos dois trabalhos do nosso grupo de pesquisa podem explicar a alta prevalência de IU e disfunção do assoalho pélvico em mulheres com diabetes mellitus (DM) gestacional. Como o músculo esquelético é o órgão-alvo principal do metabolismo de glicose, o entendimento dos mecanismos básicos envolvendo a miopatia diabética tem enorme importância para o desenvolvimento de estratégias terapêuticas para melhorar a qualidade de vida das gestantes diabéticas e reduzir os custos da assistência à saúde. Os achados desses dois modelos evidenciam que o diabetes na prenhez de ratas lesa o músculo uretral de forma similar.

Pesquisas recentes apontam que não apenas o músculo mas também a matriz extracelular (MEC) uretral desempenham importante papel no mecanismo da continência urinária ${ }^{7,8}$.
Assim, após verificar que a literatura ainda é controversa na discussão do comportamento da MEC na gênese da IU e nos efeitos do diabetes e gestação, o objetivo desta revisão foi estudar as alterações da matriz extracelular diante do diabetes e seu impacto sobre a continência urinária.

Foi realizada revisão da literatura no site PubMed de artigos dos últimos cinco anos publicados na íntegra com os seguintes descritores: collagen, diabetes, extracellular matrix, urethra, urinary incontinence.

\section{Caracterização fisiológica da matriz extracelular}

Além do componente muscular estriado e liso, a parede da uretra é composta por tecido conjuntivo especializado para cada região, como na região da lâmina própria urotelial, entre as células do tecido muscular liso e entre as células musculares estriadas do esfíncter uretral. É de extrema importância conhecer a composição, distribuição e organização dos componentes da MEC dessas diferentes regiões do tecido uretral e as modificações existentes na condição de IU, gestação e diabetes visando contribuir para um melhor entendimento das alterações uretrais diante dessas condições.

O tecido conjuntivo é constituído por fibroblastos e uma complexa rede de macromoléculas denominada matriz extracelular. A MEC contém colágeno, elastina, fibronectina, laminina e proteoglicanos que, em conjunto, conferem propriedades bioquímicas e biofísicas específicas aos diferentes tecidos 9 . É importante ressaltar que a MEC constitui uma rede tridimensional ao redor de todas as células, órgãos e tecidos do corpo. Em geral, a MEC forma um filtro biofísico para proteção e nutrição, bem como meio para facilitar a resposta imune e regeneração tecidual ${ }^{10}$. Corresponde a $20 \%$ do nosso peso corporal e trata-se de um ambiente dinâmico formado por componentes fibrilares e um gel viscoso constituído de macromoléculas altamente hidratadas ${ }^{11}$.

Os componentes estruturais da MEC, como colágeno e fibras elásticas, proporcionam rigidez mecânica e flexibilidade ao tecido, servindo também como substrato para adesão e migração celular ${ }^{12}$. Além disso, as proteoglicanas (PGs) e glicosaminoglicanas (GAGs) da MEC atuam cooperativamente aos componentes fibrilares para regular os processos de proliferação, adesão, migração, diferenciação e apoptose celular ${ }^{13}$. As moléculas da MEC também formam a membrana basal (que separa o epitélio ou endotélio do estroma), a qual é constituída principalmente por colágeno IV, lamininas, nidogem e perlecan, que auxilia na manutenção da arquitetura e integridade tecidual do trato genito-urinário ${ }^{14}$.

O sistema colágeno consiste de diferentes tipos de fibras colágenas, fibras reticulares, colágenos de ancoragem e colágenos associados a fibrilas. Já o sistema elástico apresenta fibras finas e ramificadas, formando uma rede 
bastante irregular ${ }^{10}$. O colágeno é o maior componente da MEC; existem 28 tipos de colágeno descritos em todo o corpo, mas os mais abundantes no assoalho pélvico são os tipos I e III ${ }^{15}$. O colágeno tipo I é o maior colágeno de formação fibrilar. Ele polimeriza nas fibras maiores e é o determinante primário de força tensional. O colágeno tipo III predomina nos vasos sanguíneos e está relacionado com flexibilidade e distensibilidade dos tecidos ${ }^{16}$.

As GAGs são heteropolissacarídeos, formados por unidades dissacarídicas repetitivas compostas de uma hexosamina e um açúcar não aminado, com exceção do queratam sulfato, que apresenta uma galactose substituindo o ácido urônico. As GAGs mais comuns são condroitin sulfato (CS), dermatan sulfato (DS), heparan sulfato (HS), queratan sulfato (QS), heparina $(\mathrm{H})$ e ácido hialurônico $(\mathrm{AH}$ - único que não está ligado covalentemente ao esqueleto proteico das PGs e não apresenta grupamento sulfato em sua cadeia $)^{17}$. Em razão de seu caráter polianiônico, as GAGs são capazes de interagir com uma variedade de componentes presentes tanto na MEC quanto na superfície celular. Assim, esses polímeros podem inibir ou regular a passagem de outras moléculas através da membrana basal, controlar o acesso de macromoléculas (fatores de crescimento e hormônios) à superfície celular, afetar o crescimento, migração, adesão e a diferenciação celular ${ }^{18}$.

A eficiência do colágeno é influenciada pelas metaloproteinases de matriz (MMPs). Atualmente, são descritos cerca de 23 tipos de MMPs que atuam no processo de remodelação dos componentes da matriz extracelular e, portanto, auxiliam na manutenção da homeostasia tecidual. Elas são endopeptidases dependentes de metais, principalmente zinco e cálcio. Possuem a capacidade de degradar os componentes da MEC, como colágeno, elastina, laminina, fibronectina e proteoglicanos ${ }^{19}$. A atividade e a produção das MMPs são controladas por inibidores endógenos conhecidos como inibidores teciduais de metaloproteinases ou TIMPs ${ }^{20}$.O desequilíbrio entre a ação das MMPs e dos TIMPs pode resultar em diversas condições patológicas, por exemplo, artrite reumatóide e doenças cardiovasculares $^{21}$, e na progressão dos tumores ${ }^{22,23}$.

\section{Matriz extracelular e incontinência urinária}

A IU de esforço resulta tipicamente da disfunção do mecanismo de fechamento do esfíncter uretral e/ou dos tecidos circundantes que fazem a uretra não resistir ao fluxo de urina nos períodos de aumento da pressão intra-abdominal ${ }^{24}$. Diversas pesquisas indicam que a MEC uretral desempenha papel crítico na patogênese da $\mathrm{IU}^{7,8}$.

A literatura descreve diferenças na composição e na quantidade de colágeno em mulheres com e sem IU. Alguns autores relacionaram IU com diminuição na quantidade de colágeno. Rechberger et al. ${ }^{25}$ observaram uma redução significativa de $20 \%$ no colágeno total na fáscia pubocervical em mulheres com IU. Liapis et al. ${ }^{26,27}$ também verificaram diminuição no colágeno tipo I e III na fáscia vaginal e ligamento uterosacrais de mulheres com IU e prolapso. Em 2007, Trabucco et al. ${ }^{28}$ e Song et al. ${ }^{29}$ sugeriram um remodelamento no tecido conjuntivo na região periuretral de pacientes com IU, com diminuição e distribuição anormal das fibras colágenas, resultando em uma matriz mais flexível. Todos esses autores concluíram que o colágeno tem importante papel na manutenção da continência urinária, porém o mecanismo envolvido ainda não está claro.

Por outro lado, muitos autores relacionam a IU com aumento na quantidade de colágeno periuretral em mulheres ${ }^{30-32}$. Em 1998, Falconer et al..$^{32}$ verificaram, em biópsia uretral, que a concentração de colágeno e o diâmetro das fibrilas colágenas eram 30\% maior no grupo de mulheres incontinentes e concluíram que a IU está associada com mudanças no metabolismo do colágeno, levando ao aumento na sua concentração e no tamanho de suas fibras. Essas alterações podem resultar em uma MEC mais rígida e prejudicar sua função mecânica ${ }^{32}$. Fitzgerald et al. ${ }^{31}$ também encontraram diferenças morfológicas na ultraestrutura uretral em biópsias de mulheres incontinentes. Houve presença de padrão degenerativo, desorganização nas fibras colágenas e aumento na quantidade de proteoglicanos na superfície do colágeno em algumas pacientes com IU ${ }^{31}$. Em razão da diversidade nos métodos de detecção, tipo de biópsia e avaliação dos desfechos, um consenso ainda não foi alcançado. Além disso, as amostras ainda são heterogênias e em pequeno número para permitir conclusões satisfatórias ${ }^{20}$.

Com relação às GAGs, Feldner et al. ${ }^{33}$ verificaram que mulheres incontinentes apresentaram maior quantidade total de GAGs sulfatadas e dermatan sulfato no tecido periuretral. Os autores hipotetizaram que a IU pode estar relacionada com mudanças bioquímicas nos componentes da matriz extracelular do tecido conjuntivo periuretral e que as GAGs contribuem para as propriedades físicas, já que estão envolvidas na resistência e elasticidade tecidual. Por causa da interação com o colágeno, sugere-se então que as GAGs representam papel fundamental na complacência e resistência de suporte às estruturas pélvicas ${ }^{33}$.

Matriz extracelular no diabetes e incontinência urinária A relação entre DM e IU tem sido observada em diversos estudos epidemiológicos ${ }^{34-36}$, mas os mecanismos pelos quais DM leva à IU ainda são escassos. A taxa de IU entre mulheres com diabetes é de 50 a 200\% mais elevada do que entre mulheres com níveis normais de glicose ${ }^{37-39}$. Há evidência de que a IU é mais prevalente no diabetes do que outras complicações comuns associadas a essa síndrome, como retinopatia, nefropatia e neuropatia ${ }^{40,41}$. Gharaee-Kermani et al. ${ }^{42}$ sugeriram que a obesidade, o 
DM tipo 2, a fibrose no trato urinário inferior e a perda urinária estão intrinsecamente e biologicamente ligados.

O diabetes exerce efeito na expressão, organização e modificação nos componentes da MEC em diversos órgãos ${ }^{43,44}$. Essas alterações vêm sendo descritas em humanos, por exemplo, no músculo estriado esquelético ${ }^{44} \mathrm{e}$ na artéria renal ${ }^{45}$ e em ratos no tendão da cauda ${ }^{46}$, pele ${ }^{47}$, tendão de Aquiles ${ }^{48}$, próstata $^{49}$ e uretra $^{50,51}$.

A remodelação do tecido e a fibrose parecem ser uma consequência direta do diabetes. Uma vez que a fibrose interrompe a função normal de órgãos em indivíduos diabéticos, os miofibroblastos, como um dos moduladores primários da produção e volume da MEC, são alvo terapêutico potencial para a prevenção da remodelação da matriz patológica. $\mathrm{Na}$ fibrose tubulointersticial observada na nefropatia diabética, o aumento do número de miofibroblastos tem sido associado com a deposição excessiva de $\mathrm{MEC}^{52}$.

Berria et al. ${ }^{44}$ observaram aumento significativo de colágeno tipos I e III em biópsia muscular do vasto lateral de pacientes com DM tipo 2, concluindo que mudanças na composição da MEC são características do músculo insulino-resistente. É possível que essas alterações na matriz do músculo esquelético sejam uma resposta à inflamação crônica, que leva à patologia mitocondrial e resulta em acúmulo de lipídio intracelular e resistência à insulina. Porém, esse cenário é apenas especulativo e mais estudos são necessários para explicar esse mecanismo ${ }^{44}$.

Estudos experimentais evidenciam aumento na deposição de colágeno no trato urinário inferior em animais diabéticos. Ratas após 6-8 semanas com diabetes induzido por streptozotocin (glicemia maior que $300 \mathrm{mg} / \mathrm{dL}$ ), após distensão vaginal, apresentaram aumento na deposição de colágeno entre as fibras do músculo estriado uretral. Os autores concluíram que o acúmulo de espécies reativas de oxigênio e os produtos finais de glicação avançada podem contribuir para as causas miopáticas e neuropáticas das disfunções do trato urinário inferior e que o diabetes está associado com incontinência mais severa e com atraso na recuperação das injúrias nos mecanismos da continência urinária ${ }^{50}$.
Rodrigues et al..$^{51}$ analisaram as alterações estruturais da MEC uretral em ratos machos Wistar com diabetes induzido por aloxana. Foi verificado que a atrofia muscular e a fibrose uretral ocorreram após 44 semanas e que esse processo foi mais severo no grupo diabético do que nos animais não diabéticos. Assim, as mudanças estruturais na matriz extracelular uretral são relevantes e devem ser consideradas nos estudos do trato urinário ${ }^{51}$.

Ahmed et al. ${ }^{53}$ encontraram deficiência na síntese de colágeno I e III nos tendões de ratos diabéticos, sugerindo produção desregulada das metaloproteinases. A expressão proteica de MMP-13 foi maior nos tendões de ratos diabéticos, entretanto essa diferença não foi observada na análise de expressão correspondente. Inversamente, a expressão gênica de MMP-3 foi menor nos tendões de ratos diabéticos ${ }^{53}$. A discrepância entre os resultados da expressão gênica e proteica pode ser explicada pela regulação da transcrição ou pós-tradução influenciada, potencialmente, pelos níveis elevados de glicose no diabete ${ }^{54}$.

Notou-se que as alterações presentes no músculo e na matriz extracelular uretral estão entre as principais causas de incontinência urinária de esforço. O diabetes é um dos principais causadores dessas complicações, mas ainda há controvérsias nas consequências da gestação nesse mecanismo. Os dados sugerem que a incontinência pode ser a consequência mais comum da hiperglicemia comparada a outras complicações. Identificar os fatores de risco para o desenvolvimento da IU em diabéticas é o maior objetivo na prevenção dessa condição tão comum. Enquanto a predisposição genética, paridade e idade têm um importante papel no desenvolvimento da IU de esforço, o impacto de fatores de risco modificáveis, como o DM, implica que utilizando estratégias preventivas e terapêuticas pode-se reduzir as taxas de IU e melhorar a qualidade de vida das mulheres, especialmente na gestação e no pós-parto e reduzir os custos da assistência à saúde.

\section{Agradecimentos}

Este estudo teve suporte financeiro da Fundação de Amparo à Pesquisa do Estado de São Paulo (FAPESP) (2010/11703-4 e 2010/10740-3).

\section{Referências}

1. Barbosa AM, Dias A, Marini G, Calderon IM, Witkin S, Rudge MV. Urinary incontinence and vaginal squeeze pressure two years post-cesarean delivery in primiparous women with previous gestational diabetes mellitus. Clinics (São Paulo). 2011 ; 66(8):1341-6.

2. Barnes AS. The epidemic of obesity and diabetes: trends and treatments. Tex Heart Inst J. 2011 ; 38(2): 142-4.

3. Rosenstock J, Wilson C, Fleck P. Alogliptin versus glipizide monotherapy in elderly type 2 diabetes mellitus patients with mild hyperglycaemia: a prospective, double-blind, randomized, 1-year study. Diabetes Obes Metab. 2013;15(10):906-14.

4. Wilson L, Brown JS, Shin GP, Luc KO, Subak LL. Annual direct cost of urinary incontinence. Obstet Gynecol. 2001 ;98(3):398-406.

5. Marini G, Barbosa AM, Damasceno DC, Matheus SM, Castro RA, Girão $M$, et al. Morphological changes in the fast vs slow fiber profiles of the urethras of diabetic pregnant rats. Urogynaecologia. 2011;25(1):e9:31-6. 
6. Piculo F, Marini G, Barbosa AM, Damasceno DC, Matheus SM, Felisbino SL, et al. Urethral striated muscle and extracellular matrix morphological characteristics among mildly diabetic pregnant rats: translational approach. Int Urogynecol J. 2014;25(3):403-15.

7. Chen B, Yeh J. Alterations in connective tissue metabolism in stress incontinence and prolapse. J Urol. 2011;186(5):1768-72.

8. Li GY, Cui WS, Zhou F, Gao ZZ, Xin H, Liu T, et al. Pathology of urethral fibromuscular system related to parturition-induced stress urinary incontinence and TGF-betal/Smad pathway. Mol Cell Biochem. 2012;364(1-2):329-35.

9. Hynes RO. The extracellular matrix: not just pretty fibrils. Science. 2009;326(5957): 1216-9.

10. Noguera R, Nieto OA, Tadeo I, Fariñas F, Alvaro T. Extracellular matrix, biotensegrity and tumor microenvironment. An update and overview. Histol Histopathol. 2012;27(6):693-705.

11. Stevens A, Lowe J, editors. Histología humana. 3a ed. Madrid: Elsevier Mosby; 2006. Células de sostén y la matriz extracelular; p. 46-64.

12. de Carvalho HF, Taboga SR, Vilamaior PS. Collagen type VI is a component of the extracellular matrix microfibril network of the prostatic stroma. Tissue Cell. 1997;29(2):163-70.

13. Huxley-Jones J, Pinney JW, Archer J, Robertson DL, Boot-Handford RP. Back to basics - how the evolution of the extracellular matrix underpinned vertebrate evolution. Int J Exp Pathol. 2009;90(2):95-100.

14. Byron A, Humphries JD, Humphries M. Defining the extracellular matrix using proteomics. Int J Exp Pathol. 2013;94(2):75-92.

15. Chen CC, Hijaz A, Drazba JA, Damaser MS, Daneshgari F. Collagen remodeling and suburethral inflammation might account for preserved anti-incontinence effects of cut polypropylene sling in rat model. Urology. 2009;73(2):415-20.

16. Broughton $G$ 2nd, Janis JE, Attinger CE. The basic science of wound healing. Plast Reconstr Surg. 2006; 11 (1Suppl 7):12S-34S.

17. Hay E. Cell-matrix interaction in the embryo: cell shape, cell surface, cell skeleton and their role in differentiation. In: Trelstad RL (ed). The role of extracellular matrix in development. New York: Alan R Liss; 1984. p. 1-31.

18. Cechowska-Pasko M, Palka J. Age-dependent changes in glycosaminoglycan content in the skin of fasted rats. A possible mechanism. Exp Toxicol Pathol. 2000;52(2):127-31.

19. Stamenkovic I. Matrix metalloproteinases in tumor invasion and metastasis. Semin Cancer Biol. 2000;10(6):415-33.

20. Campeau L, Gorbachinsky I, Badlani GH, Andersson KE. Pelvic floor disorders: linking genetic risk factors to biochemical changes. BJU Int. $2011 ; 108(8): 1240-7$.

21. Elkington PT, O'Kane CM, Friedland JS. The paradox of matrix metalloproteinases in infectious disease. Clin Exp Immunol. 2005; 142(1):12-20.

22. Huang S, Van Arsdall M, Tedjarati S, McCarty M, Wu W, Langley $R$, et al. Contributions of stromal metalloproteinase-9 to angiogenesis and growth of human ovarian carcinoma in mice. J Natl Cancer Inst. 2002;94(15):1134-42.

23. Wallard M, Pennington CJ, Veerakumarasivam A, Burtt G, Mills IG, Warren A, et al. Comprehensive profiling and localisation of the matrix metalloproteinases in urothelial carcinoma. $\mathrm{Br} J$ Cancer. 2006;94(4):569-77

24. Rovner ES, Ginsberg DA, Raz S. The UCLA surgical approach to sphincteric incontinence in women. World J Urol. 1997;15(5):280-94.

25. Rechberger T, Postawski K, Jakowicki JA, Gunja-Smith Z, Woessner $\mathrm{JF} \mathrm{Jr}$. Role of fascial collagen in stress urinary incontinence. Am J Obstet Gynecol. 1998;179(6 Pt 1):1511-4.
26. Liapis A, Bakas $P$, Pafiti A, Hassiakos D, Frangos-Plemenos $M$, Creatsas $G$. Changes in the quantity of collagen type I in women with genuine stress incontinence. Urol Res. 2000;28(5):323-6.

27. Liapis A, Bakas P, Pafiti A, Frangos-Plemenos M, Arnoyannaki N, Creatsas $G$. Changes of collagen type III in female patients with genuine stress incontinence and pelvic floor prolapse. Eur J Obstet Gynecol Reprod Biol. $2001 ; 97(1): 76-9$.

28. Trabucco E, Soderberg M, Cobellis L, Torella M, Bystrom B, Ekman-Ordeberg $G$, et al. Role of proteoglycans in the organization of periurethral connective tissue in women with stress urinary incontinence. Maturitas. 2007;58(4):395-405

29. Song $Y$, Hong $X, Y u Y$, Lin $Y$. Changes of collagen type III and decorin in paraurethral connective tissue from women with stress urinary incontinence and prolapse. Int Urogynecol J Pelvic Floor Dysfunct. 2007;18(12):1459-63.

30. Morley R, Cumming J, Weller R. Morphology and neuropathology of the pelvic floor in patients with stress incontinence. Int Urogynecol J Pelvic Floor Dysfunct. 1996;7(1):3-12.

31. Fitzgerald MP, Mollenhaver J, Hale DS, Benson JT, Brubaker L. Urethral collagen morphologic characteristics among women with genuine stress incontinence. Am J Obstet Gynecol. 2000; 182(6): 1565-74.

32. Falconer $C$, Blomgren $B$, Johansson $O$, Ulmsten $U$, Malmstrom $A$, Westergren-Thorsson $G$, et al. Different organization of collagen fibrils in stress-incontinent women of fertile age. Acta Obstet Gynecol Scand. 1998;77(1):87-94.

33. Feldner PC Jr, Kati LM, Sartori MG, Baracat EC, Rodrigues de Lima $G$, Nader HB, et al. Sulfated glycosaminoglycans of the periurethral tissue in women with and without stress urinary incontinence, according to genital prolapse stage. Eur J Obstet Gynecol Reprod Biol. 2006; 126(2):250-4.

34. Kim C, McEwen LN, Sarma AV, Piette JD, Herman WH. Stress urinary incontinence in women with a history of gestational diabetes mellitus. J Womens Health (Larchmt). 2008;17(5):783-92.

35. Danforth KN, Townsend MK, Curhan GC, Resnick NM, Grodstein F. Type 2 diabetes mellitus and risk of stress, urge and mixed urinary incontinence. J Urol. 2009;181(1):193-7.

36. Ebbesen MH, Hannestad YS, Midthjell K, Hunskaar S. Diabetes and urinary incontinence - prevalence data from Norway. Acta Obstet Gynecol Scand. 2007;86(10):1256-62.

37. Jackson RA, Vittinghoff E, Kanaya AM, Miles TP, Resnick HE, Kritchevsky $S B$, et al. Urinary incontinence in elderly women: findings from the Health, Aging, and Body Composition Study. Obstet Gynecol. 2004;104(2):301-7.

38. Lifford KL, Curhan GC, Hu FB, Barbieri RL, Grodstein F. Type 2 diabetes mellitus and risk of developing urinary incontinence. J Am Geriatr Soc. 2005;53(1 1):1851-7.

39. Jackson SL, Scholes D, Boyko EJ, Abraham L, Fihn SD. Urinary incontinence and diabetes in postmenopausal women. Diabetes Care. $2005 ; 28(7): 1730-8$.

40. Phelan S, Grodstein F, Brown JS. Clinical research in diabetes and urinary incontinence: what we know and need to know. J Urol. 2009; 182(Suppl 6):S14-7.

41. Phelan S, Kanaya AM, Subak LL, Hogan PE, Espeland MA, Wing $R R$, et al. Prevalence and risk factors for urinary incontinence in overweight and obese diabetic women: action for health in diabetes (look ahead) study. Diabetes Care. 2009;32(8):1391-7.

42. Gharaee-Kermani M, Rodriguez-Nieves JA, Mehra R, Vezina CA, Sarma AV, Macoska JA. Obesity-induced diabetes and lower urinary tract fibrosis promote urinary voiding dysfunction in a mouse model. Prostate. 2013;73(10):1123-33. 
43. Law B, Fowlkes V, Goldsmith JG, Carver W, Goldsmith EC. Diabetesinduced alterations in the extracellular matrix and their impact on myocardial function. Microsc Microanal. 2012;18(1):22-34.

44. Berria R, Wang L, Richardson DK, Finlayson J, Belfort R, Pratipanawatr $T$, et al. Increased collagen content in insulin-resistant skeletal muscle. Am J Physiol Endocrinol Metab. 2006;290(3):E560-5.

45. Chan DT, Watts GF, Irish AB, Ooi EM, Dogra GK. Insulin resistance and the metabolic syndrome are associated with arterial stiffness in patients with chronic kidney disease. Am J Hypertens. 2013;26(9): $1155-61$

46. Odetti P, Aragno I, Rolandi R, Garibaldi S, Valentini S, Cosso L, et al. Scanning force microscopy reveals structural alterations in diabetic rat collagen fibrils: role of protein glycation. Diabetes Metab Res Rev. 2000; 16(2):74-81.

47. Knas M, Niczyporuk M, Zalewska A, Car H. The unwounded skin remodeling in animal models of diabetes types 1 and 2. Physiol Res. 2013;62(5):519-26.

48. de Oliveira RR, Martins CS, Rocha YR, Braga AB, Mattos $R M$, Hecht $F$, et al. Experimental diabetes induces structural, inflammatory and vascular changes of Achilles tendons. PLoS One. 2013;8(10):e74942.
49. Ribeiro DL, Taboga SR, Goes RM. Diabetes induces stromal remodelling and increase in chondroitin sulphate proteoglycans of the rat ventral prostate. Int J Exp Pathol. 2009;90(4):400-1 1.

50. Kim JH, Huang X, Liu G, Moore C, Bena J, Damaser MS, et al. Diabetes slows the recovery from urinary incontinence due to simulated childbirth in female rats. Am J Physiol Regul Integr Comp Physiol. 2007;293(2):R950-5.

51. Rodrigues AA Jr, Suaid HJ, Fazan VP, Reis RB, Cologna AJ, Tucci $\mathrm{S} \mathrm{Jr}$, et al. Histologic study of urethral extracellular matrix and collagen from aging and long-term alloxan-induced diabetic male rats. Urology. $2011 ; 77(2): 510 . e 6-11$

52. Li JH, Wang W, Huang XR, Oldfield M, Schmidt AM, Cooper ME, et al. Advanced glycation end products induce tubular epithelialmyofibroblast transition through the RAGE-ERK1/2 MAP kinase signaling pathway. Am J Pathol. 2004; 164(4):1389-97.

53. Ahmed AS, Schizas N, Li J, Ahmed M, Ostenson CG, Salo P, et al. Type 2 diabetes impairs tendon repair after injury in a rat model. J Appl Physiol. 2012;113(11):1784-91.

54. Loots MA, Lamme EN, Zeegelaar J, Mekkes JR, Bos JD, Middelkoop E. Differences in cellular infiltrate and extracellular matrix of chronic diabetic and venous ulcers versus acute wounds. The J Invest Dermatol. 1998; 111 (5):850-7. 